\title{
Propagation of Electric Dipole Radiation through a Medium
}

\author{
Xin Li ${ }^{1}$ and Henk F. Arnoldus ${ }^{2}$ \\ ${ }^{1}$ Department of Physics, Millersville University, P.O. Box 1002, Millersville, PA 17551, USA \\ ${ }^{2}$ Department of Physics and Astronomy, Mississippi State University, P.O. Drawer 5167, Mississippi, MS 39762-5167, USA
}

Correspondence should be addressed to Henk F. Arnoldus, hfa1@msstate.edu

Received 23 September 2012; Accepted 11 October 2012

Academic Editors: O. Frazão and V. Matejec

Copyright (C 2012 X. Li and H. F. Arnoldus. This is an open access article distributed under the Creative Commons Attribution License, which permits unrestricted use, distribution, and reproduction in any medium, provided the original work is properly cited.

When electromagnetic energy propagates through a material medium, the paths of energy flow may be altered, as compared to propagation in free space. We consider radiation emitted by an electric dipole, embedded in a medium with permittivity $\varepsilon_{r}$ and permeability $\mu_{r}$. For a linear dipole in free space, the field lines of energy flow are straight, but when the imaginary part of $\varepsilon_{r}$ is finite, the field lines in the material become curves in the near field of the dipole. Therefore, the energy flow is redistributed due to the damping in the material. For a circular dipole in free space, the field lines of energy flow wind around the axis perpendicular to the plane of rotation of the dipole moment. When $\varepsilon_{r}$ has an imaginary part, this flow pattern is altered drastically. Furthermore, when the real part of $\varepsilon_{r}$ is negative, the direction of rotation of the flow lines reverses. In that case, the energy in the field rotates opposite to the direction of rotation of the dipole moment. It is indicated that in metamaterials with a negative index of refraction this may lead to an observable effect in the far field.

\section{Introduction}

When optical radiation from a localized source is observed at a large distance, it appears as if the light travels along straight lines. Similarly, light scattered or reflected by an object seems to travel from the object to an observer along straight lines, known as optical rays. These lines are the flow lines of the energy in the radiation field. In close vicinity of the source, however, these flow lines are in general curves, and intricate field line patterns may appear. Such structures can be found when the flow of radiation is resolved on a scale smaller than a wavelength. Particularly interesting is the possible presence of singularities and optical vortices. The first prediction of the existence of an optical vortex was made by Braunbek and Laukien in 1952 [1]. They considered the diffraction of a plane wave around the edge of a conducting half plane, and they found that a vortex should appear at the illuminated side of the plane, and close to the edge. Another mechanism that may lead to singularities and vortices in the energy flow is interference. We have shown recently $[2,3]$ that when a point source is located near a reflecting surface, numerous vortices are present in the energy-flow pattern when the source is about a wavelength away from the surface. A different type of vortex in the energy-flow pattern results from a rotation inside the source. We shall show in the next section that radiation emitted by an electric dipole may have such a vortex structure [4], and such vortices appear in multipole radiation of any order [5].

A much more subtle effect is the redirection of energy flow when the radiation passes through a material medium. A material will in general absorb radiation along its path of propagation, but we shall show that the presence of material will in general also lead to a redistribution of the power flow. It will be shown that in media the field lines curve due to the presence of the media, and that vortices which are present due to a rotation in the source are altered dramatically by the embedding medium.

\section{Electric Dipole Radiation in Free Space}

The most important source of electromagnetic radiation is arguably the oscillating electric dipole. When the current density in a localized source oscillates harmonically with 


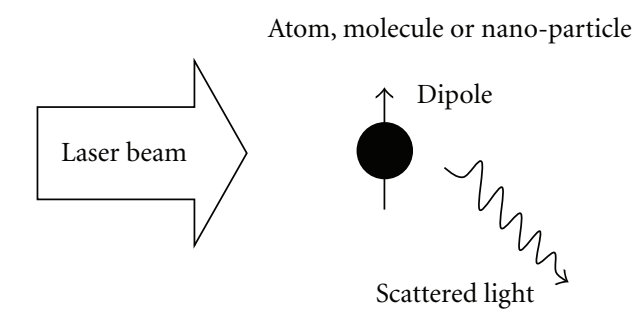

Figure 1: Setup for a nano-particle in a laser beam.

angular frequency $\omega$, it has an electric dipole moment of the form

$$
\mathbf{d}(t)=\operatorname{Re}\left(\mathbf{d} e^{-i \omega t}\right),
$$

where $\mathbf{d}$ is the complex amplitude. When the dimension of the source is small compared to the wavelength of the light, then electric dipole radiation gives the dominant contribution to the emitted radiation (compared to higher order multipoles). For example, when a small particle, like an atom, a molecule or a nanoparticle, is irradiated by a laser of angular frequency $\omega$, then the emitted, or scattered, radiation will be electric dipole radiation, at least to a very good approximation. The setup is illustrated in Figure 1.

The emitted electric field will have the form

$$
\mathbf{E}(\mathbf{r}, t)=\operatorname{Re}\left[\mathbf{E}(\mathbf{r}) e^{-i \omega t}\right],
$$

with $\mathbf{E}(\mathbf{r})$ the complex amplitude, and the magnetic field $\mathbf{B}(\mathbf{r}, t)$ can be represented in a similar form. The Poynting vector is defined as

$$
\mathbf{S}(\mathbf{r})=\frac{1}{2 \mu_{\mathrm{o}}} \operatorname{Re}\left[\mathbf{E}(\mathbf{r})^{*} \times \mathbf{B}(\mathbf{r})\right] .
$$

This is the time-averaged Poynting vector, in which terms that oscillate at twice the optical frequency have been dropped, since these average to zero on a time scale of an optical cycle. The complex amplitudes of the electric and magnetic fields of an oscillating dipole are well known [6], and this Poynting vector can readily be constructed.

The field lines of energy flow are the field lines of the vector field $\mathbf{S}(\mathbf{r})$. When we indicate by $\mathbf{r}$ a point on a field line, then the curve can be parametrized as $\mathbf{r}(u)$, where $u$ is a dummy variable. At any point along the field line, the vector $\mathbf{S}(\mathbf{r})$ must be on the tangent line, and therefore the field lines $\mathbf{r}(u)$ are solutions of the autonomous differential equation

$$
\frac{\mathrm{d} \mathbf{r}}{\mathrm{d} u}=f(\mathbf{r}) \mathbf{S}(\mathbf{r}) .
$$

Here, $f(\mathbf{r})$ can be any positive function of $\mathbf{r}$ since a field line pattern only depends on the directions of the vectors of the vector field, and not on their magnitude. A common choice for the function $f(\mathbf{r})$ is $1 /|\mathbf{S}(\mathbf{r})|$, so that the right-hand side of (4) becomes a unit vector, and the parameter $u$ is equal to the arc length measured along a field line. Each numerical step size $\Delta u$ is then a step $\Delta u$ along the field line, and this more or less guarantees an equal spacing of numerical data

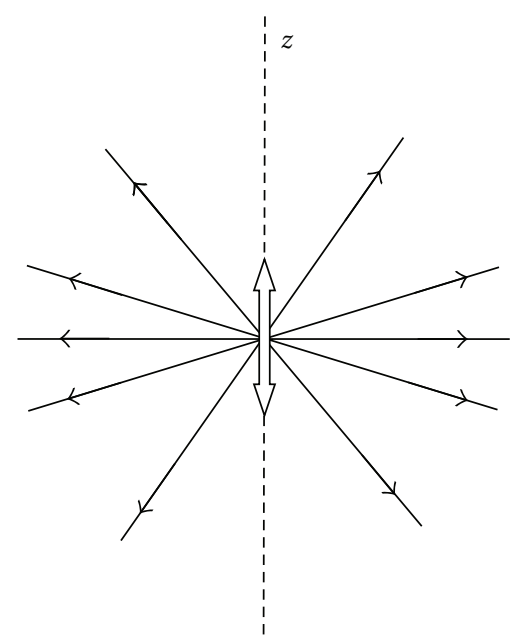

FIGURE 2: Field lines of the Poynting vector for the radiation emitted by a linear dipole in free space.

along each field line. Through each point $\left(x_{0}, y_{0}, z_{0}\right)$ in space we have a field line, and (4) can be integrated numerically, given this starting point $\left(x_{0}, y_{0}, z_{0}\right)$.

Let us first consider a linear dipole moment for which $\mathbf{d}=d_{\mathrm{o}} \mathbf{e}_{z}$ with $d_{\mathrm{o}}>0$. Then the dipole moment $\mathbf{d}(t)$ oscillates harmonically along the $z$ axis, and the Poynting vector is found to be

$$
\mathbf{S}(\mathbf{r})=\frac{3 P_{\mathrm{o}}}{8 \pi r^{2}} \widehat{\mathbf{r}}_{\sin ^{2}} \theta,
$$

with $(r, \theta, \phi)$ the spherical coordinates with respect to the $z$ axis. The constant $P_{\mathrm{o}}$ is defined as

$$
P_{\mathrm{o}}=\frac{c k_{\mathrm{o}}^{4}}{12 \pi \varepsilon_{\mathrm{o}}} d_{\mathrm{o}}^{2}
$$

with $k_{\mathrm{o}}=\omega / c$, and this $P_{\mathrm{o}}$ equals the total power emitted by the dipole. We see from (5) that $\mathbf{S}(\mathbf{r})$ is proportional to the radial unit vector $\widehat{\mathbf{r}}$ for all field points, and therefore the field lines of $\mathbf{S}(\mathbf{r})$ are straight lines, emanating from the location of the dipole. The field line pattern is shown in Figure 2.

A linear dipole moment is induced when a particle is illuminated by a linearly polarized laser, as in the setup in Figure 1, and the oscillation direction is along the direction of the polarization of the beam. When this laser is leftcircular polarized then the electric field vector of the beam rotates counterclockwise when looking into the oncoming beam. If we take the propagation direction as the $z$ axis, then this electric field rotates counterclockwise in the $x y$ plane when viewed down the positive $z$ axis. Then the complex amplitude of the induced dipole moment is

$$
\mathbf{d}=-\frac{d_{\mathrm{o}}}{\sqrt{2}}\left(\mathbf{e}_{x}+i \mathbf{e}_{y}\right)
$$

and the dipole moment itself is

$$
\mathbf{d}(t)=-\frac{d_{\mathrm{o}}}{\sqrt{2}}\left[\mathbf{e}_{x} \cos (\omega t)+\mathbf{e}_{y} \sin (\omega t)\right] .
$$




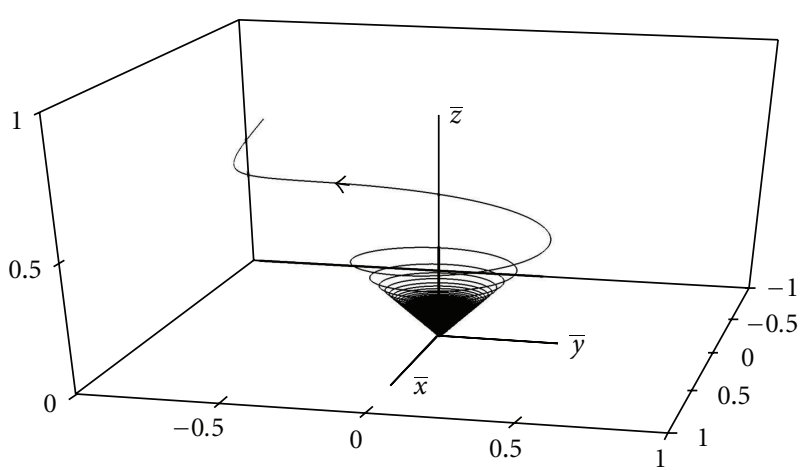

Figure 3: A typical field line of the Poynting vector for the radiation emitted by a circular dipole in free space.

This dipole moment rotates counterclockwise in the $x y$ plane, so it has the same direction of rotation as the incident beam. The Poynting vector is found to be

$$
\mathbf{S}(\mathbf{r})=\frac{3 P_{\mathrm{o}}}{8 \pi r^{2}}\left[\left(1-\frac{1}{2} \sin ^{2} \theta\right) \hat{\mathbf{r}}+\frac{1}{q}\left(1+\frac{1}{q^{2}}\right) \mathbf{e}_{\phi} \sin \theta\right],
$$

where we have set $q=k_{0} r$ for the dimensionless distance between the dipole and the field point $\mathbf{r}$. In units of $q$, a distance of $2 \pi$ corresponds to one optical wavelength. Interestingly, (4) for the field lines can be solved in closed form for this simple system $[4,7]$. The term proportional to $\hat{\mathbf{r}}$ in (9) corresponds to power flowing into the radially outward direction, but now a term proportional to $\mathbf{e}_{\phi}$ appears. This contribution gives rise to a rotation of the field lines around the $z$ axis. A typical field line is shown in Figure 3. The variables on the axes are $\bar{x}=k_{0} x$, and so forth. It can be shown that each field line lies on a cone around the $z$ axis, and a field line spirals around the $z$ axis while staying on this cone. We see from the figure that the spatial extent of the vortex structure is well below a wavelength. At a large distance from the source, as compared to a wavelength, the term containing $\mathbf{e}_{\phi}$ vanishes, and each field line approaches a straight line. Figure 4 shows several field lines on the same cone, and the figure clearly gives the impression of energy swirling around the $z$ axis in the near field.

Figure 5 shows a field line for the radiation emitted by a rotating dipole, as seen from far away. The field line approaches asymptotically the straight line $\ell$, and we see that it appears as if the line came from a point in the $x y$ plane, indicated by vector $\mathbf{q}_{d}$, which is displaced with respect to the location of the dipole. Therefore, when viewed from the far field, it seems that the position of the dipole is shifted as a result of the rotation of the field lines in the near field [8]. The actual image of the source is a result of the observation of a bundle of field lines, rather than a single line, and a more detailed analysis shows that the rotation of the field lines indeed results in a shift of the image [9-11]. This shift has been observed experimentally [12].

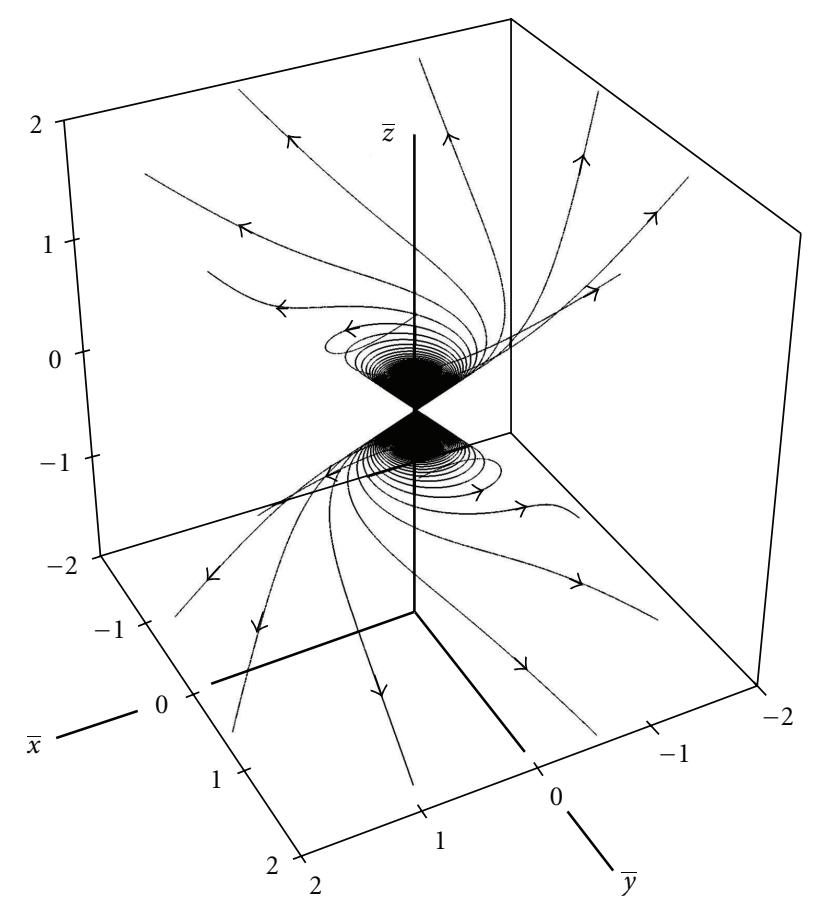

FIGURE 4: Energy flow pattern in the near field of a circular dipole in free space.

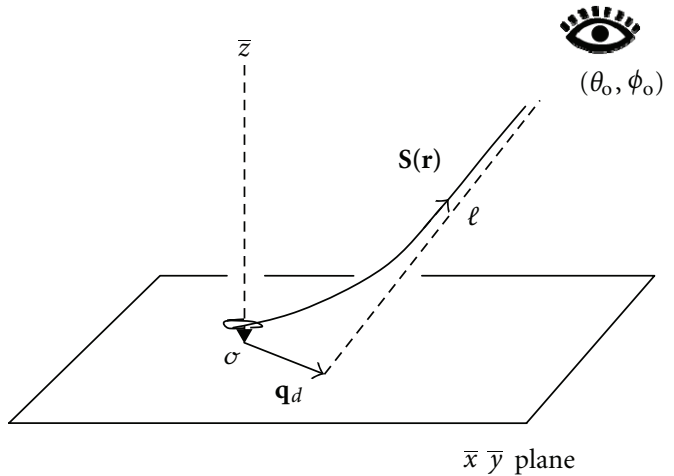

Figure 5: Due to the rotation near the source, a field line seems to be displaced when viewed from the far field.

\section{Electric Dipole Radiation in a Medium}

The electromagnetic properties of a material are represented by the relative permittivity $\varepsilon_{r}$ and the relative permeability $\mu_{r}$. Both are complex, in general, with a nonnegative imaginary part, and they depend on the angular frequency $\omega$. The index of refraction $n$ of the material is a solution of

$$
n^{2}=\varepsilon_{r} \mu_{r}
$$

and we take the solution with

$$
\operatorname{Im}(n) \geq 0 .
$$


For an oscillating electric dipole embedded in this medium, the complex amplitudes of the emitted electric and magnetic fields are

$$
\begin{aligned}
\mathbf{E}(\mathbf{r})= & -\frac{1}{3 \varepsilon_{\mathrm{o}} \varepsilon_{r}} \mathbf{d} \delta(\mathbf{r})+\mu_{r} \frac{k_{\mathrm{o}}^{3}}{4 \pi \varepsilon_{\mathrm{o}}} \\
& \times\left\{\mathbf{d}-(\hat{\mathbf{r}} \cdot \mathbf{d}) \hat{\mathbf{r}}+[\mathbf{d}-3(\hat{\mathbf{r}} \cdot \mathbf{d}) \hat{\mathbf{r}}] \frac{i}{n q}\left(1+\frac{i}{n q}\right)\right\} \\
& \times \frac{e^{i n q}}{q}, \\
& \mathbf{B}(\mathbf{r})=\frac{n \mu_{r}}{c} \frac{k_{\mathrm{o}}^{3}}{4 \pi \varepsilon_{\mathrm{o}}}(\hat{\mathbf{r}} \times \mathbf{d})\left(1+\frac{i}{n q}\right) \frac{e^{i n q}}{q}
\end{aligned}
$$

The first term on the right-hand side of (12) is the self field, which only exists inside the point source. The remaining terms are proportional to $\exp (i n q)$, and these terms represent outgoing spherical waves.

The Poynting vector in a medium is defined as

$$
\mathbf{S}(\mathbf{r})=\frac{1}{2 \mu_{\mathrm{o}}} \operatorname{Re}\left[\frac{1}{\mu_{r}} \mathbf{E}(\mathbf{r})^{*} \times \mathbf{B}(\mathbf{r})\right] .
$$

First we define the vector field $\boldsymbol{\sigma}(\mathbf{q})$ by

$$
\mathbf{S}(\mathbf{r})=\left|\mu_{r}\right|^{2} \frac{3 P_{\mathrm{o}}}{8 \pi r^{2}} e^{-2 q \operatorname{Im} n} \boldsymbol{\sigma}(\mathbf{q}) .
$$

The overall factor which is split off is positive, and therefore the field lines of $\boldsymbol{\sigma}(\mathbf{q})$ are the same as the field lines of $\mathbf{S}(\mathbf{r})$. The field $\boldsymbol{\sigma}(\mathbf{q})$ is dimensionless and it only depends on the dimensionless representation $\mathbf{q}$ of the field point where $\boldsymbol{\sigma}(\mathbf{q})$ is evaluated. The factor $\exp (-2 q \operatorname{Im} n)$ gives an exponential decay of the magnitude of the Poynting vector along a field line when $\operatorname{Im} n>0$, and this corresponds to absorption in the material. The direction of the Poynting vector is unaffected by this absorption, since it is an overall positive factor. We introduce the complex unit vector $\mathbf{u}$ by

$$
\mathbf{d}=d_{\mathrm{o}} \mathbf{u}, \quad d_{\mathrm{o}}>0, \quad \mathbf{u} \cdot \mathbf{u}^{*}=1 .
$$

From (12) and (13), we obtain

$$
\begin{aligned}
\boldsymbol{\sigma}(\mathbf{q})= & {\left[1-\left(\hat{\mathbf{r}} \cdot \mathbf{u}^{*}\right)(\hat{\mathbf{r}} \cdot \mathbf{u})\right] \widehat{\mathbf{r}} \operatorname{Re}\left[\frac{n}{\mu_{r}}\left(1+\frac{i}{n q}\right)\right] } \\
+ & \left|1+\frac{i}{n q}\right|^{2} \frac{1}{|n|^{2} q} \\
& \times\left\{\left[1-3\left(\hat{\mathbf{r}} \cdot \mathbf{u}^{*}\right)(\hat{\mathbf{r}} \cdot \mathbf{u})\right] \widehat{\mathbf{r}} \operatorname{Im}\left(\varepsilon_{r}\right)\right. \\
& \left.+2 \operatorname{Im}\left[\varepsilon_{r}\left(\hat{\mathbf{r}} \cdot \mathbf{u}^{*}\right) \mathbf{u}\right]\right\} .
\end{aligned}
$$

In the far field, where $r$ is much larger than an optical wavelength, we have $q=k_{\mathrm{o}} r \gg 1$, and (17) simplifies to

$$
\boldsymbol{\sigma}(\mathbf{q}) \approx\left[1-\left(\hat{\mathbf{r}} \cdot \mathbf{u}^{*}\right)(\hat{\mathbf{r}} \cdot \mathbf{u})\right] \widehat{\mathbf{r}} \operatorname{Re}\left(\frac{n}{\mu_{r}}\right) .
$$

The Poynting vector is approximately proportional to $\widehat{\mathbf{r}}$, which is radially outward. Therefore, in the far field the field lines of energy flow asymptotically approach a straight line, as in Figure 5. Consequently, any curving of the field lines can only occur in the near field, for example, within a few wavelengths of the dipole. Further inspection of the righthand side of (17) shows that all terms are proportional to $\widehat{\mathbf{r}}$, except the term containing $\operatorname{Im}\left[\varepsilon_{r}\left(\hat{\mathbf{r}} \cdot \mathbf{u}^{*}\right) \mathbf{u}\right]$. Therefore, any curving of field lines is due to this factor.

\section{Linear Dipole in a Medium}

We now consider a linear dipole, oscillating along the $z$ axis, so we have $\mathbf{u}=\mathbf{e}_{z}$. Equation (17) simplifies to

$$
\begin{aligned}
\boldsymbol{\sigma}(\mathbf{q})= & \hat{\mathbf{r}} \sin ^{2} \theta \operatorname{Re}\left[\frac{n}{\mu_{r}}\left(1+\frac{i}{n q}\right)\right] \\
& +\left|1+\frac{i}{n q}\right|^{2} \frac{1}{|n|^{2} q} \\
& \times\left[\hat{\mathbf{r}}\left(1-3 \cos ^{2} \theta\right)+2 \mathbf{e}_{z} \cos \theta\right] \operatorname{Im} \varepsilon_{r} .
\end{aligned}
$$

For a linear dipole in free space, $\varepsilon_{r}, \mu_{r}$, and $n$ equal unity, and (19) becomes $\boldsymbol{\sigma}(\mathbf{q})=\widehat{\mathbf{r}} \sin ^{2} \theta$, as in (5). Vector $\boldsymbol{\sigma}(\mathbf{q})$ is proportional to $\hat{\mathbf{r}}$ at all field points, and therefore the field lines are straight and run radially outward, as illustrated in Figure 2 . The only term not proportional to $\hat{\mathbf{r}}$ in (19) is the term containing $\mathbf{e}_{z} \cos \theta \operatorname{Im} \varepsilon_{r}$. This term is proportional to $\mathbf{e}_{z}$, and results in a bending of the field lines into the $z$ direction. Figure 6 depicts the resulting field line pattern. A more detailed analysis [13] shows that near the $z$ axis, the field lines approach the $z$ axis under $90^{\circ}$, and stop there. Therefore the $z$ axis is a singular line of the flow pattern. In free space, near the $z$ axis the field lines run parallel to the $z$ axis whereas in a medium they are perpendicular to the $z$ axis. Field lines that start at the dipole and stop at the $z$ axis represent nonradiative energy flow in the sense that energy transported along these curves does not end up in the far field.

The term responsible for the bending of the field lines is proportional to $\operatorname{Im} \varepsilon_{r}$, so this phenomenon only appears when $\operatorname{Im} \varepsilon_{r}>0$. Since $\operatorname{Im} \varepsilon_{r}>0$ leads to dissipation of energy upon propagation, we conclude that the curving of the flow lines is a result of damping in the material. Interestingly, an imaginary part of $\mu_{r}$ does not have this effect.

\section{Rotating Dipole in a Medium}

In this section we consider the rotating dipole, with $\mathbf{d}$ given by (7). Working out the right-hand side of (17) yields

$$
\begin{gathered}
\boldsymbol{\sigma}(\mathbf{q})=\left(1-\frac{1}{2} \sin ^{2} \theta\right) \hat{\mathbf{r}} \operatorname{Re}\left[\frac{n}{\mu_{r}}\left(1+\frac{i}{n q}\right)\right]+\left|1+\frac{i}{n q}\right|^{2} \\
\times \frac{1}{|n|^{2} q}\left\{\left[\left(1-\frac{1}{2} \sin ^{2} \theta\right) \hat{\mathbf{r}}+\frac{1}{2} \sin (2 \theta) \mathbf{e}_{\theta}\right] \operatorname{Im} \varepsilon_{r}\right. \\
\left.+\mathbf{e}_{\phi}(\sin \theta) \operatorname{Re} \varepsilon_{r}\right\} .
\end{gathered}
$$




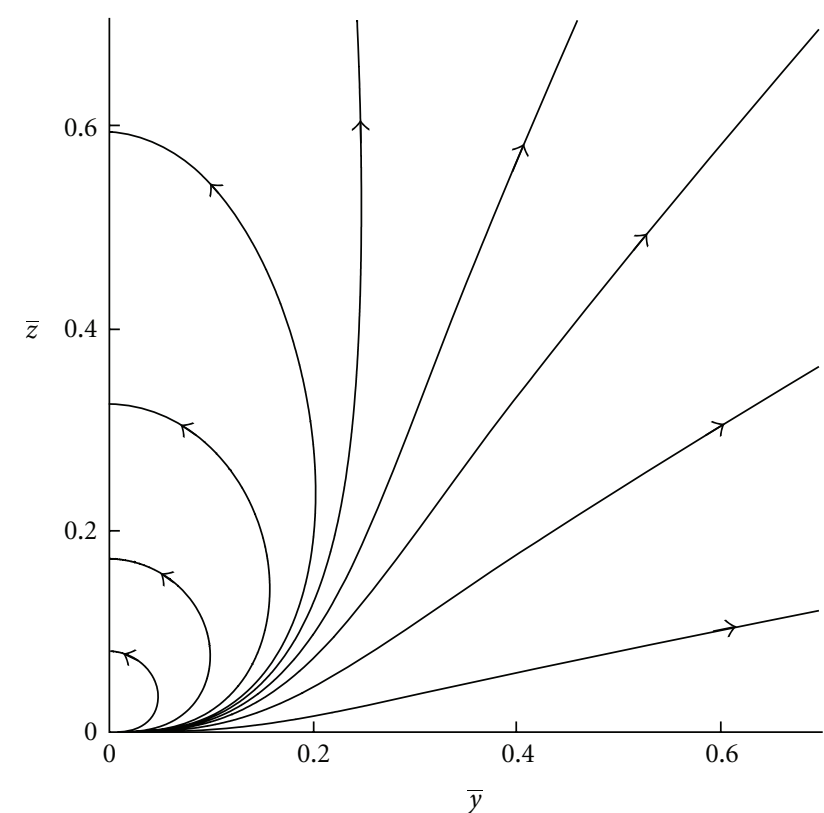

Figure 6: Shown are field lines of the Poynting vector for a dipole oscillating along the $z$ axis. The parameters are $\varepsilon_{r}=1.7+0.06 i$ and $\mu_{r}=1$, which are the values for water at $3 \mu \mathrm{m}$. The index of refraction is $n=1.3+0.023 i$.

It can be shown that

$$
\operatorname{Re}\left[\frac{n}{\mu_{r}}\left(1+\frac{i}{n q}\right)\right] \geq 0
$$

for any $\varepsilon_{r}$ and $\mu_{r}$, so the first term on the right-hand side corresponds to radial power flow, and this is the only term that survives in the far field. The last term is proportional to $\mathbf{e}_{\phi}$, and this gives rise to rotation of field lines around the $z$ axis. If the imaginary part of $\varepsilon_{r}$ would be zero, the remaining terms would vanish, and the field lines would wind around the $z$ axis and lie on a cone, as in Figures 3 and 4. The term containing $\mathbf{e}_{\phi}$ is proportional to $\operatorname{Re} \varepsilon_{r}$. For ordinary dielectrics, this is positive, but for other materials this may be negative. In that case, the field lines rotate around the $z$ axis opposite to the rotation direction of the dipole moment.

When the imaginary part of $\varepsilon_{r}$ is positive, a term with $\mathbf{e}_{\theta}$ appears, and consequently the field lines do not lie on a cone anymore. Figure 7 shows two typical field lines, and Figure 8 shows the corresponding field lines in free space. We notice that due to the damping, the cone has become a funnel, and the field lines are not as densely wound around the $z$ axis. Even though the imaginary part of $\varepsilon_{r}$ is very small for the illustration in Figure 7, the effect is dramatic. The rotation of the field lines near the source leads to a displacement of the dipole image in the far field, as in Figure 5 [14]. Just as for the dipole in free space, this provides a possible method for experimental observation of this phenomenon. As for the linear dipole, we find here also that the imaginary part of $\varepsilon_{r}$ leads to a redistribution of the energy flow in the near field.

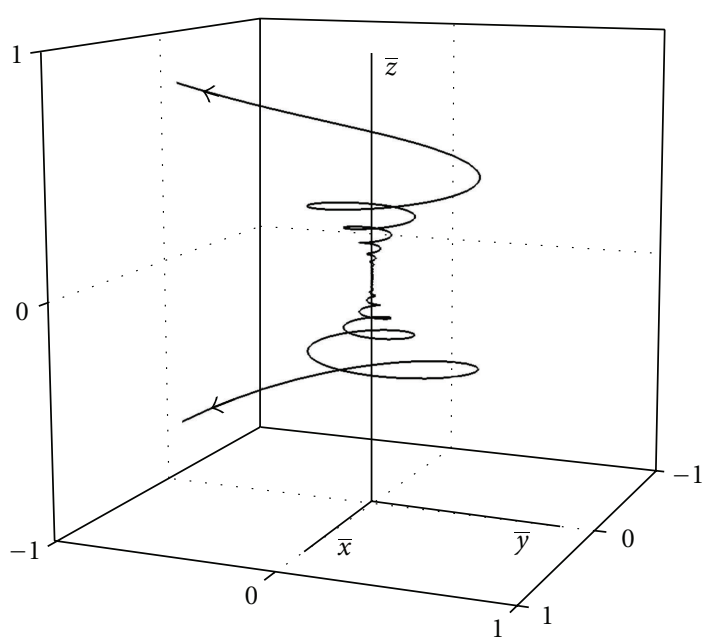

Figure 7: Shown are two field lines for a circular dipole in a dielectric with $\varepsilon_{r}=1+0.07 i$ and $\mu_{r}=1$.

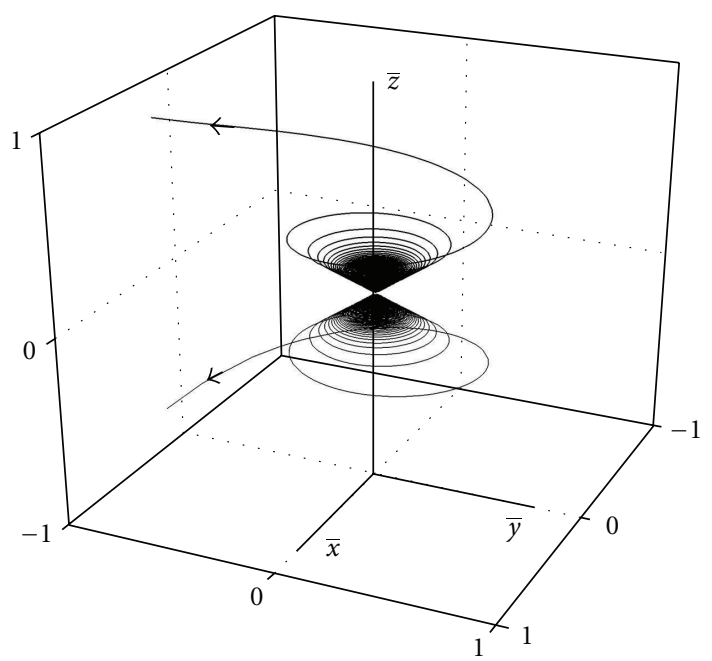

FIGURE 8: Shown are two field lines for a circular dipole in free space. The dipole rotates counterclockwise when viewed down the positive $z$ axis, and the field lines wind around the $z$ axis in the same direction.

\section{Dipole in a Negative Index of Refraction Material}

For most natural materials, the value of $\mu_{r}$ is very close to unity. For dielectrics, the real part of $\varepsilon_{r}$ is positive, and the imaginary part of $\varepsilon_{r}$ is usually very small. For metals, the real part of $\varepsilon_{r}$ can be positive (in the UV) or negative (visible and below), and the imaginary part of $\varepsilon_{r}$ is relatively small. If the real part of $\varepsilon_{r}$ is positive and the imaginary part is small, then the index of refraction $n$ is approximately positive, and the material is transparent. When $\operatorname{Re} \varepsilon_{r}<0, n$ is approximately positive imaginary, and light cannot propagate through the medium. Most waves are evanescent and do not reach the far field. 
There is, however, no physical reason for any limitations on the values of $\varepsilon_{r}$ and $\mu_{r}$, except that their imaginary parts cannot be negative (for causality reasons). If we could have a material for which both $\varepsilon_{r}$ and $\mu_{r}$ would be almost negative, at a certain frequency, then it follows from (10) and (11) that the index of refraction is negative. Such a medium would be transparent, even though it would have the appearance of a metal. However, it appears that such materials do not occur in nature. Metamaterials are artificially structured composites, consisting of arrays of subwavelength structures. Their electromagnetic response is not only determined by the material from which they are constructed, but also by the geometry of the design. Since the dimension of the structures is well below a wavelength, the material can be represented as a continuum, and the electromagnetic properties can be accounted for by $\varepsilon_{r}$ and $\mu_{r}$. Of particular interest are materials for which the real parts of both $\varepsilon_{r}$ and $\mu_{r}$ are negative, and for which the imaginary parts of both $\varepsilon_{r}$ and $\mu_{r}$ are small. These materials have a negative index of refraction. It was shown by Veselago [15] that such materials would have very peculiar properties. In particular, upon refraction through an interface with such a material, an optical ray is transmitted at the opposite side of the normal to the surface, as compared to refraction by a dielectric medium. This is a result of the fact that in such a material the Poynting vector is opposite to the direction of the phase velocity.

Let us assume that $\varepsilon_{r}$ and $\mu_{r}$ are both positive or both negative. Then $n$ has the same sign as $\varepsilon_{r}$ and $\mu_{r}$, and (17) simplifies to [16]

$$
\begin{aligned}
\boldsymbol{\sigma}(\mathbf{q})= & \frac{n}{\mu_{r}}\left[1-\left(\widehat{\mathbf{r}} \cdot \mathbf{u}^{*}\right)(\hat{\mathbf{r}} \cdot \mathbf{u})\right] \widehat{\mathbf{r}} \\
& +\left(1+\frac{1}{n^{2} q^{2}}\right) \frac{2}{\mu_{r} q} \operatorname{Im}\left(\hat{\mathbf{r}} \cdot \mathbf{u}^{*}\right) \mathbf{u}
\end{aligned}
$$

For a linear dipole embedded in this medium, vector $\mathbf{u}$ is real, and the second term on the right-hand side vanishes. Therefore, $\boldsymbol{\sigma}(\mathbf{q})$ is proportional to $\hat{\mathbf{r}}$, and the field lines are straight as in Figure 2. For a circular dipole, the second term contributes, and this gives the rotations in the field lines. The only difference between a material with $n>0$ and $n<0$ is the sign of $\mu_{r}$ in this term. For a dielectric, this sign is positive, and the field lines rotate counterclockwise in the $x y$ plane, when viewed down the $z$ axis, just as in free space. For a negative index of refraction material, the sign of $\mu_{r}$ is negative, and therefore this reverses the direction of rotation as compared to free space or a dielectric. Figure 8 shows two field lines for a circular dipole in free space, and Figure 9 for a circular dipole in a negative index of refraction material. We see from Figure 9 that the field lines rotate around the $z$ axis opposite to the rotation direction of the dipole. It was shown in the previous section that the direction of rotation is determined by the sign of $\operatorname{Re} \varepsilon_{\mathrm{r}}$. In the derivation of (22) from (17) we have used the fact that $\varepsilon_{r}$ and $\mu_{r}$ have the same sign. So the reversal of rotation direction is a result of $\operatorname{Re} \varepsilon_{r}<0$ for the case of a metamaterial with negative index of refraction.

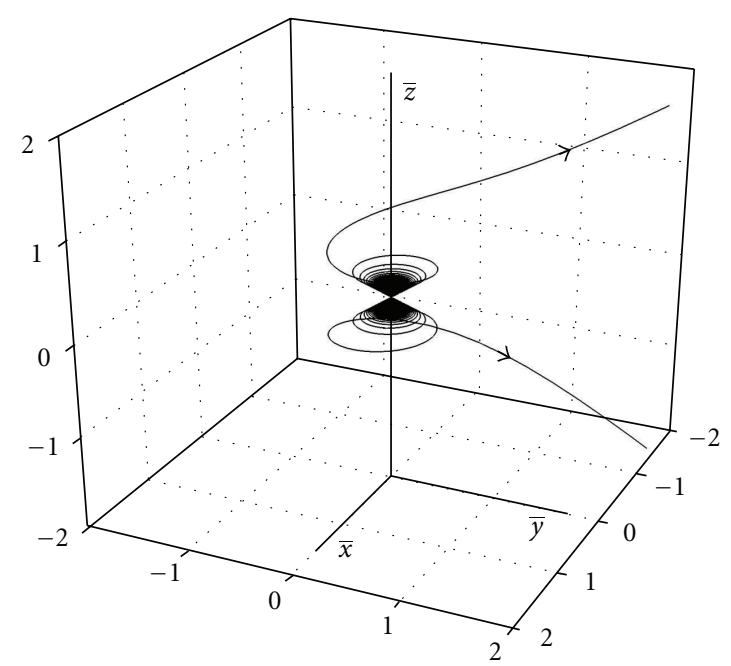

FIgURE 9: Two field lines for a circular dipole in a negative index of refraction material. The dipole rotates counterclockwise when viewed down the positive $z$ axis, and the field lines wind around the $z$ axis in the opposite direction.

\section{Conclusions}

The flow lines of electromagnetic energy are the field lines of the Poynting vector. Far away from a localized source, an interface or any obstacles, these field lines approach asymptotically straight lines. Near a source or any structure in the field, these field lines are usually curves, and intricate flow patterns of energy may appear. This can be a result of diffraction, interference, or a rotation in the source itself. A much less obvious mechanism for the curving of field lines is the presence of an embedding medium for the source. We have considered the field lines of energy flow for an electric dipole, and we have compared the flow patterns in free space to the flow patterns in an embedding medium. For a linear dipole, the field lines in free space are straight lines, as shown in Figure 2, but in an embedding medium these field lines curve as in Figure 6. The curving appears due to the imaginary part of the dielectric constant $\varepsilon_{r}$, and hence is a result of damping in the material. It was shown that already a very small imaginary part has dramatic effects in the optical near field, as can be seen from Figure 6. Near the dipole axis (the $z$ axis in the figure), the field lines approach this line under $90^{\circ}$, whereas in free space the field lines run parallel to this axis. The semiloops in the figure represent energy flow from the dipole to the $z$ axis, and this energy does not propagate to the far field. Therefore, as a result of the damping, some energy stays in the near field, where it is absorbed by the medium. It can also be seen from the figure that the field lines come out of the dipole in a direction perpendicular to the axis, whereas in free space the energy leaves the dipole under any angle. The imaginary part of $\varepsilon_{r}$ does not only result in absorption upon propagation, but it leads to a redistribution of the energy flow as well.

For a rotating dipole moment in free space, the field lines of energy flow swirl around the $z$ axis (which is perpendicular to the plane of rotation of the dipole moment) numerous 
times, before asymptotically approaching a straight line, as shown in Figures 3-5. Each field line lies on a cone around the $z$ axis. When this dipole is embedded in a medium, this field line pattern changes, and some typical field lines are shown in Figure 7. It can be shown that each field line leaves the dipole along the $z$ axis, whereas in free space the field lines come out of the dipole in any direction (the angle of the cone). The cone changes to a funnel shape, and the density of the winding pattern of each field line diminishes considerably. This change is again due to the imaginary part of $\varepsilon_{r}$. Interestingly, the imaginary part of the permeability $\mu_{r}$, which also accounts for absorption, appears to have no effect.

A metamaterial is an artificial medium, which can have a combination of values of $\varepsilon_{r}$ and $\mu_{r}$ that do not occur in nature. Of particular interest are materials for which the real parts of $\varepsilon_{r}$ and $\mu_{r}$ are both negative. Such materials have a negative index of refraction, if the absorption is relatively small, and these materials are transparent. The ultimate goal is to artificially construct a material with $n=-1$ for a given frequency. When a linear dipole is located in such a medium, the flow lines of energy are identical to the flow lines in free space. However, for a rotating dipole moment, the direction of rotation of the field lines around the $z$ axis reverses, as compared to the direction of rotation in free space. It was shown that this effect occurs for any material for which the real part of $\varepsilon_{r}$ is negative, so this is not really a unique property of negative index of refraction materials. However, natural materials with $\operatorname{Re} \varepsilon_{r}<0$ usually have $\mu_{r} \approx 1$, and therefore the index of refraction is imaginary. Such materials do not support propagating modes, and even though the field lines reverse direction, this effect only appears in the near field inside the material, and is most likely not observable. For a negative index of refraction material, the energy flowing along the reversed field lines travels to the far field, and is amenable to direct experimental observation. The image of the dipole will shift, as indicated in Figure 5, but this shift is in the opposite direction as for emission in free space.

\section{References}

[1] W. Braunbek and G. Laukien, "Einzelheiten zur HalbebenenBeugung," Optik, vol. 9, pp. 174-179, 1952.

[2] X. Li, J. Shu, and H. F. Arnoldus, "Optical vortices and singularities due to interference in atomic radiation near a mirror," Optics Letters, vol. 34, no. 22, pp. 3595-3597, 2009.

[3] X. Li and H. F. Arnoldus, "Electric dipole radiation near a mirror," Physical Review A, vol. 81, no. 5, Article ID 053844, 10 pages, 2010.

[4] H. F. Arnoldus and J. T. Foley, "The dipole vortex," Optics Communications, vol. 231, no. 1-6, pp. 115-128, 2004.

[5] H. F. Arnoldus, "Vortices in multipole radiation," Optics Communications, vol. 252, no. 4-6, pp. 253-261, 2005.

[6] J. D. Jackson, Classical Electrodynamics, Wiley, New York, NY, USA, 3rd edition, 1999.

[7] J. Shu, X. Li, and H. F. Arnoldus, "Energy flow lines for the radiation emitted by a dipole," Journal of Modern Optics, vol. 55, no. 15, pp. 2457-2471, 2008.

[8] H. F. Arnoldus, X. Li, and J. Shu, "Subwavelength displacement of the far-field image of a radiating dipole," Optics Letters, vol. 33, no. 13, pp. 1446-1448, 2008.
[9] X. Li, J. Shu, and H. F. Arnoldus, "Far-field detection of the dipole vortex," Optics Letters, vol. 33, no. 19, pp. 2269-2271, 2008.

[10] J. Shu, X. Li, and H. F. Arnoldus, "Nanoscale shift of the intensity distribution of dipole radiation," Journal of the Optical Society of America A, vol. 26, no. 2, pp. 395-402, 2009.

[11] X. Li and H. F. Arnoldus, "Macroscopic far-field observation of the sub-wavelength near-field dipole vortex," Physics Letters, Section A, vol. 374, no. 8, pp. 1063-1067, 2010.

[12] D. Haefner, S. Sukhov, and A. Dogariu, "Spin hall effect of light in spherical geometry," Physical Review Letters, vol. 102, no. 12, Article ID 123903, 4 pages, 2009.

[13] X. Li, D. M. Pierce, and H. F. Arnoldus, "Redistribution of energy flow in a material due to damping," Optics Letters, vol. 36, no. 3, pp. 349-351, 2011.

[14] X. Li, D. M. Pierce, and H. F. Arnoldus, "Damping of the dipole vortex," Journal of the Optical Society of America A, vol. 28, no. 5, pp. 778-785, 2011.

[15] V. G. Veselago, "The electrodynamics of substances with simultaneously negative values of $\varepsilon$ and $\mu$," Soviet Physics Uspekhi, vol. 10, no. 4, pp. 509-514, 1968.

[16] X. Li and H. F. Arnoldus, "Reversal of the dipole vortex in a negative index of refraction material," Physics Letters, Section A, vol. 374, no. 43, pp. 4479-4482, 2010. 

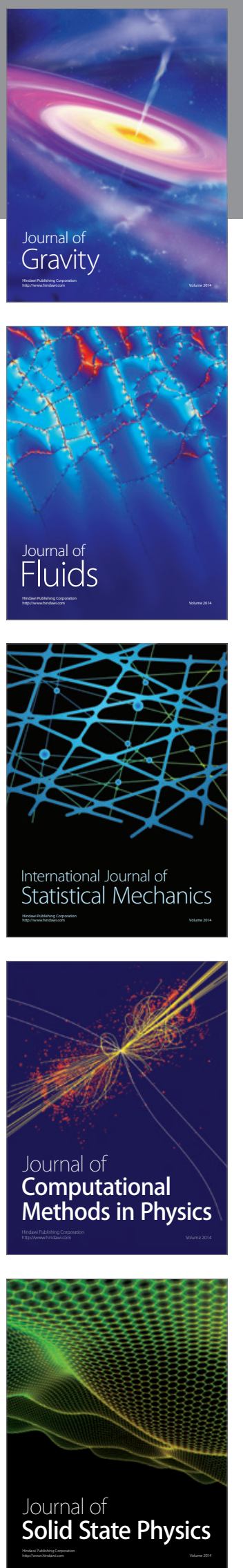
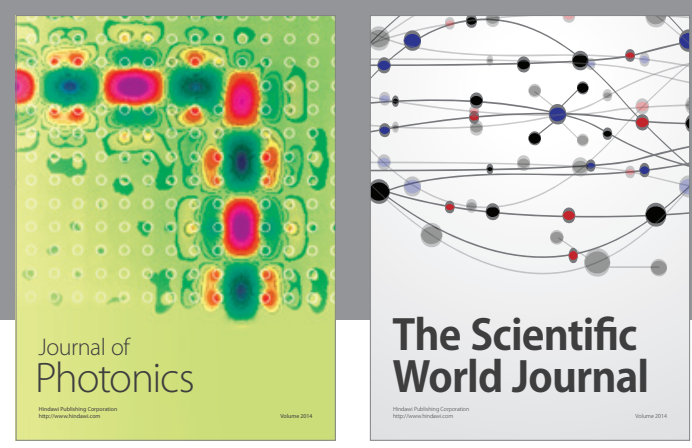

The Scientific World Journal

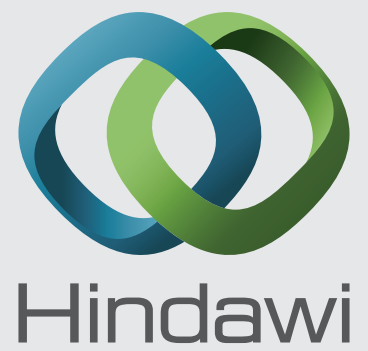

Submit your manuscripts at http://www.hindawi.com
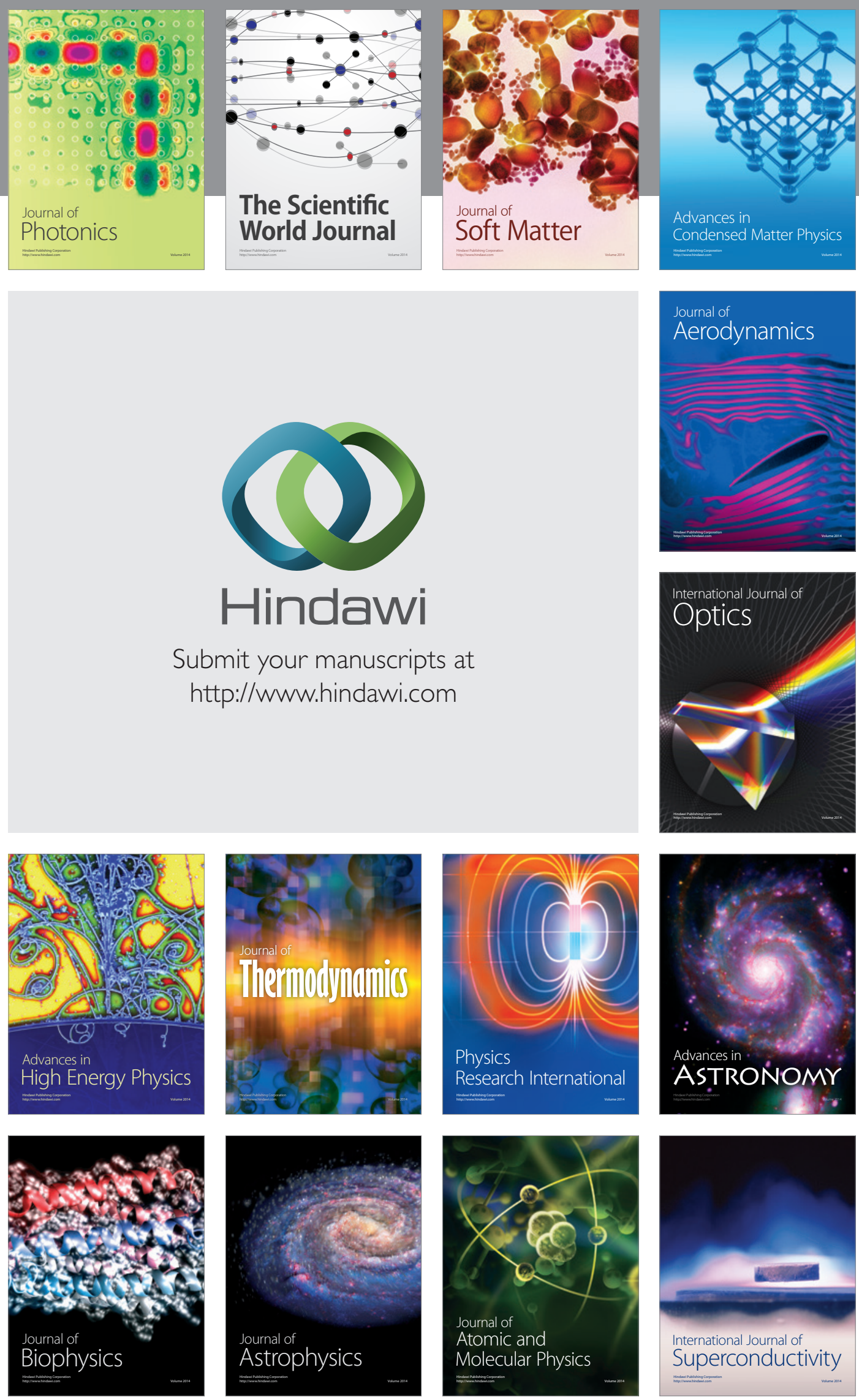
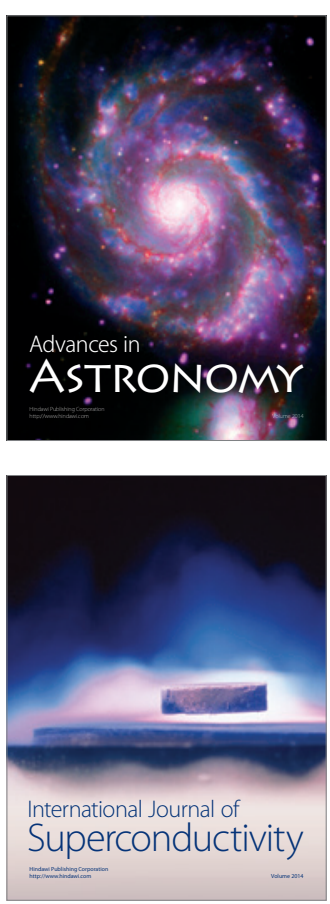